\title{
Toward a Unified Systems Engineering Education
}

\author{
Odd Andreas Asbjornsen and Robbert J. Hamann
}

\begin{abstract}
The systems engineering education is analyzed in this paper. The root of systems engineering is system theory, and both are given a brief overview. The methodology of systems engineering is used to design an educational concept. Four alternative approaches, and their merits are tested and evaluated against the requirements defined for the education. The conclusion, for the benefit both of engineering education in general and systems engineering in particular, is an integration of systems engineering principles into the education of all branches of engineering, rooted as they are in system theory and design practice already. This approach means a rationalization of the present engineering education, and meets the requirements from industry to a wider spread use of systems engineering principles and practices.
\end{abstract}

Index Terms-Engineering education, system thinking, systems engineering, systems engineering education concepts.

\section{BACKGROUND}

A $S$ TECHNICAL, societal, and economic systems become more and more complex, there is a need for integrated views and evaluations, not only of the systems themselves but also of their mutual interactions and their interaction with the environment. There is a need for education in this area, and systems engineering is an approach, a process and a discipline, which addresses the complex challenges of integrated views of large and small systems in the engineering education. But there is a dilemma. In order to master integrated views of technology a sound basis for understanding the technology is required. Traditional education in engineering meets that requirement in the various disciplines, but usually lacks the ability to cope with interdisciplinary issues. Hence there is a need to bridge this gap in the engineering education, and this paper addresses means and methods to accomplish this by various approaches to the education in systems engineering.

\section{SYSTEM THEORY}

System thinking goes back to Greek philosophers and has been a topic of philosophy ever since [11], [13]. Systems engineering applies system theory to technical systems, be it that the methodology is applicable to the design of any system in hardware, software, and bioware (human organizations) or combinations. The definition of technical systems, as proposed by modern philosophers [12], may be useful for the present purpose of this paper:

Manuscript received April 1, 1998; revised June 1, 1998 and December 1, 1998.

O. A. Asbjornsen, deceased, was with Systems Engineering, Policy Analysis, and Management, Faculty of Technology, Policy, and Management, Delft University of Technology, NL-2600 GA Delft, The Netherlands.

R. J. Hamann was with Fokker Space BV, NL 2303 DB Leiden, The Netherlands. He is now with System Integration/Space Systems Analysis and Design, Faculty of Aerospace Engineering, Delft University of Technology, NL-2629 HS Delft, The Netherlands.

Publisher Item Identifier S 1094-6977(00)04789-1.
A technical system, $S T=\langle C, A, O, R\rangle$, is a set characterized by its components $C$, the set $A$ of processes and interactions that constitute its structure, the objectives Ointended for the system, and the results $R$ that are effectively achieved. Among the components $C$ there must be a subset of intentional agents (the controllers, users, or operators of the system), that conceive of the set $O_{\text {of }}$ objectives and perform a subset of actions $(A)$ needed for the control and management of the system.

Therefore, in the design of a unified systems engineering education, a number of requirements to that education may be defined:

- knowledge shall be attained of the components $(C)$ and their functional performance;

- of the processes and interactions $(A)$ between the components, i.e., the system structure;

- of the system objectives $(O)$, what the system is supposed to achieve, i.e., the needs and requirements;

- of how to measure the system performance $(R)$, i.e., how the system performs in relation to the needs and requirements;

- of how the system design and operation shall be controlled to achieve the objectives, i.e., to meet the needs and satisfy the requirements.

An important issue left out in the philosopher's definition is the time, or life span perspective of the system. All system designs are for the future, requiring an anticipation of the life cycle events imposed on the system.

Define the Boundaries and Interfaces for the System and Its Components: Boundaries are the contours defining the region of existence of systems, its subsystems, and its elements. In a mathematical language, a boundary is usually defined as the contour enclosing the set of system elements, while the interfaces are understood as the face of contact between elements where interactions occur. It is clearly of great importance to identify the system, its boundaries and interfaces, as they are implicit in the total system functionality. In a set notation, a system description is recursive, to be used in decomposition and aggregation of the system

The set of system elements:

$$
C_{i}=C_{i-1} \cup M_{i} ; \quad C_{0}=O
$$

The set of system boundaries:

$$
B\left(C_{i}\right)=\left(B\left(C_{i-1}\right) \cup B\left(M_{i}\right)\right) \cap B(E) .
$$

Here are $M$ basic elements, $C$ a set of elements, and $B$ a set of boundaries, and $E$ the environment of an element or a set of elements; in fact, the complementary set to $C$. 
Define the System Structure: All systems have physical and functional interactions between the elements. These interactions determine the connections between elements, or its physical and functional structure. The structures, together with the functional performance of the elements in this structure, determine the total system performance

Interactions:

$$
\begin{aligned}
R\left(C, E_{S, i_{i}}\right) & =R\left(C_{i-1}, E_{S, i-1}\right) \cup R\left(M_{i}, E_{P, i}\right) ; \\
E_{S, i} & =\neg C_{i} ; \quad E_{P, i}=\neg M_{i} .
\end{aligned}
$$

Here is $R$ a set of functions, processes and interactions. Each element in a system has a specific functional performance. This is given by the nature of the elements, physical, chemical, biological, or otherwise. The nature of the element determines its functionality. However, its function is equally determined by the interactions with other elements and with the environment. Elements must be defined and analyzed in this context, which leads to the requirement of expert education in the pertinent areas of the systems. An example on such detailed knowledge is found in the generalized state equations (mathematical models) applied to system dynamics

System dynamics:

$$
\frac{d \boldsymbol{x}}{d t}=\boldsymbol{f}_{x}\left(\boldsymbol{a}_{x, x}, \boldsymbol{a}_{u, x}, \boldsymbol{a}_{d, x}, \boldsymbol{x}, \boldsymbol{u}, \boldsymbol{d}\right)
$$

Measurement dynamics:

$$
\frac{d \boldsymbol{y}}{d t}=\boldsymbol{f}_{y}\left(\boldsymbol{a}_{y, y}, \boldsymbol{a}_{x, y}, \boldsymbol{a}_{u, y}, \boldsymbol{a}_{d, y}, \boldsymbol{y}, \boldsymbol{x}, \boldsymbol{u}, \boldsymbol{d}\right)
$$

Control dynamics:

$$
\frac{d \boldsymbol{u}}{d t}=\boldsymbol{f}_{u}\left(\boldsymbol{a}_{x, u}, \boldsymbol{a}_{u, u}, \boldsymbol{a}_{d, u}, \boldsymbol{p}, \boldsymbol{x}, \boldsymbol{u}, \boldsymbol{d}\right) .
$$

Here are $f$ the set of functional structures, $\boldsymbol{x}$ the system state variables, $\boldsymbol{y}$ the system performance observations, $\boldsymbol{u}$ the control variables, $\boldsymbol{d}$ the disturbances, $\boldsymbol{p}$ the desired system performance, and $\boldsymbol{a}$ system parameters. As it is seen, this analytical characterization of system dynamics complies very well with modern philosophers' definition.

Anticipate Disturbances, Threats, and Challenges to the System in Its Lifetime: The core of systems engineering deals with the process of bringing a system into being. Therefore, all systems are designed for a future lifetime of operation. A lifetime analysis, most frequently referred to as the system life cycle assessment, of systems is absolutely essential, however quite difficult, imprecise and incomplete usually. Still, a proper understanding of the life cycle events and system exposures during its mission, short or long, is needed. For example, in economic systems the anticipated cash flow profiles for the system are essential for the justification for an investment. If the models in (3) are reasonably correct, they may serve the purpose of anticipation. Furthermore, it is seen that an anticipation of disturbances plays an important role in the evaluation of system performance.
Improving System Understanding: System understanding may be accomplished in different ways, by verbal explanations, by analogies, by logics and by mathematics, where (3) is an example. The level of understanding depends on the level and specific kind of education [3]. System analysis should adapt to those conditions, but preferably based on a set of common principles, for example the causality principle, the conservation and balance principle, the principle of intensive and extensive properties, the principle of stocks and flows, etc. In an educational context, it is clear that similarities to the system analysis are found in practically every engineering discipline. Practical experience from industrial process control has shown that the abstract concepts of systems, system thinking, and systems engineering are better accepted and appreciated once a professional education is completed, and the better the more that education is inclined to system thinking. Most people learn by experience and examples.

\section{SYSTEMS ENGINEERING}

Systems engineering is more than theory, it is also a process, the process of "bringing the system into being" [8]. In this process, there are several aspects that do not fit into, or are covered by, system theory. The most important aspect may be the functional and operational needs analysis of the system to be designed, and the mission, objectives or purpose the system is designed for, as emphasized in the system definition. This involves a definition of the stakeholders and their involvement in the system design and operation. Once the functional and operational needs are analyzed and the mission, objective or purpose of the system is agreed upon, the requirements to the system performance over its mission or lifetime are defined. In this process there are conflicting requirements, and also conflicting needs, that have to be sorted out by the stakeholders in negotiations or by some sort of tradeoff. This part of systems engineering is closely related to the general engineering design process, be it a product design or a production plant design. The process is subject to management and control, just as any other production process. The similarity in the control processes is obvious. Therefore, the systems engineering education must relate to the systems engineering process as outlined by the typical steps below [7].

Define the System Stakeholders: A system is designed for someone, used by someone, designed by someone, affecting someone, obeying the rules of someone, etc. The "someones" are the stakeholders. They are the customers who pay, the user who operates, or uses the system. They are the developers who develops, configures and implements the system, the third party that inadvertently is affected by the system, and the authorities who set the rules for the system design. A new group is added in Industrial Ecology [1] as the fourth party, which is the future generation. The stakeholders have interests and involvement in the system design.

Analyze the Functional and Operational Needs and Their Rationales: The stakeholders may have different needs for the system, often classified in functional, operational, physical, and financial needs. They may be conflicting, but they have to be expressed and analyzed up-front. Often the needs are formulated 
in a biased way, as preconceived notions to their possible satisfaction. The analysis of needs attempt to answer the question, what is needed in the sense of functional and operational capability of the system. In principle, there should be a justified purpose behind all requests and actions. A scrutiny and analysis of the rationales behind the needs will expose this. The analysis of rationales tries to answer the question, why the needs are there. The analysis often discloses preconceived notions.

Anticipate the System Mission, the Threats and Disturbances, and Assess Life Cycle Performance: The future use of the system is to complete a mission, a purpose or an objective. This must be analyzed up-front. The analysis includes the anticipation of the mission itself, possible changes, threats, and disturbances, as well as of system performance over its life cycle. These anticipations, along with the needs, form the basis for the system performance requirements.

Define the System Performance Requirements: Based on the foregoing analyses of needs, rationales, as well as the assessment of life cycle performance, the performance requirements are defined. For large systems, requirement definition is a major undertaking, for large systems with object oriented database programs, for smaller systems just lists and spreadsheets, which pin point open-ended, nonspecified, or conflicting requirements. The requirement definition attempts to answer the question, how well the system shall perform. Performance requirements are essential for the total system design specifications, as well as for the single system elements. Their definitions are also early warnings of critical issues, for example conflicts that may not be possible to resolve, or a system performance difficult or too costly to attain.

Suggest Preliminary System Concepts: The analysis of needs and rationales, assessment of life cycle performance, as well as the definition of requirements guides in the search for system concepts. This is the first entry point of technology, where the searching for system concepts attempts to answer the question, how the system may be realized, or "brought into being." Iterative tradeoff loops may screen and rank alternative concepts.

Analyze System Performance: This is the area where the system theory, system modeling, and simulation have their major impact on the system design. A design is not realized at the conceptual stage, and the only possible analyses of system performance are the ones based on models and simulations. Models and simulations use empirical data from existing and similar applications. However, it should be emphasized that there are several levels of complexity and reliability of such models, from the simplest mental models formulated verbally, via expert system models to the most advanced continuous and discrete, deterministic and stochastic mathematical models. They all have their place, relevance and application in the life span of bringing the system into being as a model based systems engineering [9].

Test and Evaluate System Performance Against Requirements: The purpose of the system performance analysis is to serve as a quality test of the system. This is the performance testing and evaluation phase, where the conceptual system performance may be compared with the requirements. Those tests are usually carried out for a combination of expected life cycle scenarios in system conditions and exposures in its different phases. The method of performance measurement, as well as the conditions, under which the measurements are valid for comparison with the requirements, needs to be agreed upon by the stakeholders.

Modify Concepts According to a Tradeoff Analysis: Deviations between performance and requirements are incentives for improvements of the system conceptual design, just like in any feedback control loop. However, since there are many requirements to be satisfied, not all can be met to the same degree. A multiobjective tradeoff, optimization or decision is usually what guides the feedback improvements.

Accept a Baseline Concept for Detailed System Design: The preliminary conceptual design obtained by the previous steps, forms the baseline concept recommended for further system development and fabrication, just like in any other engineering design process. Often it is useful to retain additional concepts as fallback for critical issues, which have been identified. Into the baseline concept are now imbedded total system design and performance evaluation in a concurrent fashion, life cycle assessment including cost and revenues, maintenance, integrated logistic life cycle support, changes and upgrade requirements, etc., as the best possible starting point for the detailed design, production, implementation, and commissioning of the system.

\section{DESIGN OF Systems ENGINEERING EDUCATION}

The basics of the systems engineering process, as outlined above, may very well be applied to the design of systems engineering education. This system has an overwhelming constituent (the students), which is governed by human factors. Both the learning and teaching processes are dominated by social and psychological factors such as cultural background, family environment, perception, motivation, confidence and imagination, etc. In addition, these factors are often highly dynamic with respect to time, the individual, and groupings of individuals. Education is a highly multidisciplinary conglomerate, which often utilizes different vocabulary, perspectives, and methods for the portrayal of similar concepts in different education areas or at different times. The introduction of a broad based education in engineering, such as systems engineering, or system thinking, as it is introduced in management [14], may seem fairly well justified on the background of requirements from industry and business. Furthermore, it is felt that major improvements may be obtained in engineering education itself by the application of systems engineering principles and practices [7], as it is here shown to benefit the design of systems engineering education.

\section{A. Education System Analysis}

Education systems are governed by some fundamental principles related to learning, teaching and information exchange. An education system analysis attempts to identify some of the characteristic parameters in those principles, as illustrated in a very simplified way below. Education systems have some vaguely identifiable variables and phenomena in it, which may affect the way education systems work. Apart from psychological factors like motivation, inspiration security, etc., there are a few obvious 
phenomena in the education system. One is related to the dynamics of learning and forgetting. Both the learning and forgetting curves may be modeled by exponential functions

Learning rate:

$$
L(t)=L_{0} \alpha e^{-\alpha t}
$$

Forgetting factor:

$$
f(t)=e^{-\beta(t-\tau)}
$$

Accumulated knowledge:

$$
K(t)=\int_{0}^{t} e^{-\alpha t} e^{-\beta(t-\tau)} d \tau=\frac{L_{0} \alpha}{\alpha-\beta}\left(e^{-\beta t}-e^{-\alpha t}\right) .
$$

Since the accumulated knowledge is the difference between what was learnt and what was forgotten, there is a set of necessary requirements to obtain a positive knowledge from the learning experience. First, the rate constant, $\beta$, of forgetting must be significantly less than the rate constant, $\alpha$, of learning. Second, there is an optimum learning period, $t_{0}$, at which the knowledge gain is optimal

Optimal learning time:

$$
t_{0}=\frac{1}{\alpha-\beta} \ln \left(\frac{\alpha}{\beta}\right) .
$$

The problem with learning and forgetting is that they are different for each individual. As a possible guideline, exponential models may be fitted to each individual by testing on factual data, and used to indicate the optimal time of learning, or an aptitude for learning in a class.

Teaching and learning are accomplished by information exchange. What is accomplished of knowledge and understanding, depends heavily on the amount and quality of information exchange. The phenomenon may be illustrated by a variable expressing the degree of confusion. If the amount of information exchange in insufficient, the confusion is high. If the amount of information exchange is too high, the degree of confusion is also high. Hence, the degree of confusion curve is a typical bathtub curve with a minimum confusion at the optimum information exchange. Such a bathtub may be modeled by the sum of two power functions

Degree of confusion curve:

$$
c=a x^{-\alpha}+b x^{\beta} .
$$

Here is $c$, a level of confusion, $x$ the amount of information exchange, $a, b, \alpha, \beta$, are parameters fitted to each individual. The optimum information exchange, $x_{0}$, is

Optimum exchange of information:

$$
x_{0}=\sqrt[\alpha+\beta]{\frac{a \alpha}{b \beta}} .
$$

The confusion characteristics represent ability to perceive information, or a concrete condition. As with learning, perception is individual, and the abilities may be tested individually. The power model above simply serves as an illustration of the phenomenon and it characteristics.

There is another fundamental phenomenon of learning by subjects, and that is the interactions between the various subjects taught. One subject may be used extensively in another, for example mathematics in physics, thermodynamics in physical chemistry, in chemical engineering, in power engineering, etc. This should be taken care of in the sequential planning of the course schedule [10].

Let the interaction and the interdependence between subjects, for example a theory and its application, be defined by an affinity coefficient, $a_{k, j}$, say between subject $k$ and $j$. If subject $k$ should come before subject $j$, then $a_{k, j}$ is positive. If the opposite is the case, $a_{k, j}$ is negative. In order to avoid knowledge loss by forgetting and confusion, the course schedule should be planned such that subjects with high affinity are close in sequence. The time difference between them, say $\Delta T_{k, j}$, is positive for positive $a_{k, j}$, and negative for negative $a_{k, j}$. A simple objective function for course scheduling could then be

Course scheduling:

$$
\min [F]=\min \left[\sum_{k=1}^{m} \sum_{j=k+1}^{m} a_{i, j} \Delta T_{i, j}\right] .
$$

The summation is taken over all subjects to be covered, and the decision variables are the subject sequence and their associated time intervals. The constraints of the optimization are the infeasible situations where there is a time collision or time overlap of subjects.

Thus, each application has a certain affinity or degree of application of a theory, or fundamental principle, given by its affinity coefficient to the theories. For example, if an application no. $j$ in Fig. 1 applies $10 \%$ of theory $k$, this may be interpreted as follows: An equivalence of $10 \%$ of the learning effort spent on application $j$ should have been spent on the preparation of theory $k$ for that application. The theory and application matrix in Fig. 1 gives a quick overview of the relative importance of the subjects. The affinities in a row indicate the significance of a particular theory for all applications in that row. The average of the affinities in the row indicates the significance of the particular theory in that row. The affinities in a column indicate the amount of the various theories required for that application. The average affinity of the entries in a column indicates the average amount of theory required for that application.

Hence, the affinity concept serves another important purpose. Going through a row of affinity numbers will show the applications where the same theory is being used. It will also indicate where a particular theory appears with the strongest impact. The result of that exercise should be a significant reduction in overlap. In the present education system, the same theory is often taught over and over in a variety of applications, sometimes with different approaches and terminology. The teaching of the application suffers from the overlap and repetition, and the understanding of the theory suffers from confusion caused by different emphasis, approaches and terminology. 


\begin{tabular}{|c|c|c|}
\hline Theory & $\rightarrow \mathrm{j}$ & \\
\hline $\begin{array}{l}\text { Appli- } \\
\text { cation }\end{array}$ & $\begin{array}{l}\text { Affinity } \\
\text { coefficient }\end{array}$ & \\
\hline$\downarrow$ & & $a_{k, j}$ \\
\hline $\mathrm{k}$ & & \\
\hline
\end{tabular}

Fig. 1. Matrix structure of knowledge.

Finally, a characteristic feature of all knowledge and learning is the dual nature of knowledge, the knowing why (understanding the underlying theory) and the knowing how (understanding the application) [4]. The problem with the combination of knowing why and knowing how, is that they are strongly interconnected in the matrix structure of the affinities, as shown in Fig. 1. Fig. 2 shows the affinity (also referred to as level of expertise) as a third dimension imposed on the affinity matrix in Fig. 1. The higher the column of affinity, the higher level of expertise is attained in the combined theory and application [5].

\section{B. Systems Engineering Methodology}

On the background of the system analysis, it is appropriate to apply the systems engineering process to design conceptual systems for systems engineering education. The methodology outlined above will be used.

Define the System Stakeholders: The stakeholders in education system design are several. The students are the prime customers, paying for their education, but they are also the users. However, there is another line of customers, those who will employ the students after education. Then there is the university faculty who both develops and uses the educational system. The so-called scientific community has an interest in the quality of education, as a standard for the scientific research, and there are the professional organizations and other authorities that set the professional standards for the education. Finally, there are general regulations for the physical and social facilities and the environment, set by the government.

Analyze the Functional and Operational Needs: The basic needs for the student stakeholders is to get a professional education which is in line with and accepted by the industry and business they will be employed by. The industry and business need graduates that are capable of contributing to the progress, quality and competitiveness of the business. This is pretty much the same needs as those of the society in general. The university needs the cash flow from the student flow, and the faculty needs an educational environment and a course program to foster cooperation with other faculty and students, to excel in their teaching, research and academic life in general.

Analyze the Rationales Behind the Needs: The rationale behind the needs of the students is fairly obvious, it concerns their future career and they trust the university knows what the professional career requirements are. These are among the reasons why they invest time and money in the university. The faculty has several reasons behind their needs: To be respected in their peer group, to enjoy the satisfaction of good teaching and to see the logical interplay between theory and practice, both in teaching and research. The rationale behind the industrial and

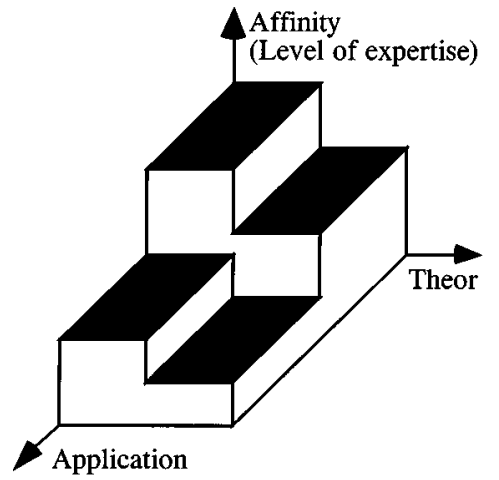

Fig. 2. Affinity or level of expertise in theory and application

societal needs is to progress and keep abreast with the most recent technology at affordable cost.

Anticipate the System Mission and Assess Life Cycle Performance: The life cycle of education systems is composed of a sequence of minor episodes, i.e., the residence time of an average student in the university environment. By the end of each of those episodes, requirements should be met to satisfy the professional requirements for the subsequent phases of the student's life cycle as a professional engineer. The life cycle for the education system is different from the life cycle of the student. Furthermore, the effects and impacts of all possible changes in technology, society emphasis, financial outlook, etc., should be included in the longer life cycle scenarios of the education system as such.

Define the System Performance Requirements: The definition of requirements to an education system is a major concern of a university, but this concern is often given insufficient attention. In the present analysis, the requirements are divided into four different categories, those related to the student stakeholders, to the business and industry, to the professional organizations and to the teachers. Therefore, the definition of requirements stands out as the most important issue.

\section{Requirements to the Systems Engineering Education}

The pressure and the initiative to take up systems engineering at a university level, has come from industry, not from the academia. The motivation from industry is that a unified approach to system theory and system thinking will benefit industry and society in a very positive way indeed. The first and foremost requirement to systems engineering education is to comply with what the professional tasks and challenges are for the profession, not only presently, but also preferably over the life cycle of the individual student. The tasks and challenges may be approached in a cooperation with the student themselves and their future customers. This may be accomplished by industrial lectures and industrially oriented projects. Below are some examples on future professional requirements [5], as applied to the engineering profession. With only small modifications those requirements may apply to any discipline.

Broad-Based Qualitative Knowledge: Future engineering jobs require ability to understand and analyze large integrated or complex systems on a qualitative multidisciplinary level. Qualitative cause and effect analyses of technical, organizational, 
and social systems based on similarity principles are required. The findings, opinions and explanations shall be communicated in a language, in images and with symbols understandable in a multidisciplinary team. The present system suffers from a division into professional sectors with limited interaction and hardly any possibilities to develop cross-disciplinary education. The ability to present projects, explain phenomena or express opinions is grossly underdeveloped. systems engineering is an attempt to bridge over this deficiency.

Deep Quantitative Knowledge: Broad-based knowledge has a tendency to be shallow. Shallow knowledge fosters professional insecurity, which is dangerous in teamwork with others when based on trust [15]. A professional platform of deep quantitative knowledge seems to be required. A shift from broad based to deep knowledge is required in an area of specialization. A professional shall be prepared to play the role of an expert in a multidisciplinary work team, but also to conduct quantitative cause and effect analysis in other fields of the engineering specialization whenever needed, or, as a minimum, to know how and where to find other expert knowledge. Similarities and analogies may help accomplish that [3]. Every profession has a set of requirements to knowledge and skill, and engineering is no exception. Shallow and mediocre knowledge in an engineering profession is a danger to the society and to the individual.

Systems Engineering Ability and Insight: It becomes more and more clear, that errors and deficiencies in large integrated systems stem from inability to overview the total system, and its environment, still without losing sight of the importance of details. In particular are those interfaces critical, where each side of the interface is covered by different disciplines. This requires an ability to understand other disciplines and to explain ones own. The top-down detailing and the bottom-up aggregation of system functions and behavior enforce insight. The root to the system behavior is the needs analysis and requirements definitions, and any engineering profession will require these skills.

Learning Ability: Learning, sharing and communicating knowledge in teams and in cooperation with others is the essence of "The Fifth Discipline" [14], which is defined as "Team Learning." The four other disciplines are defined as "Systems Thinking," "Personal Mastery," working with "Mental Models," and "Building Shared Visions." By a careful examination of engineering education, a deficiency in teamwork, sharing knowledge in cooperative learning, and building shared vision, is observed. Those disciplines are certainly not part of the academic requirements and standards in engineering, as many graduates seem to be unprepared for the dramatic change from academic work to industrial work.

Human Factors: Many of the problems facing the engineer of the twenty-first century will be related to human factors and user behavior. As such, an insight into psychology and social behavior is required together with an appreciation and understanding of human factors in technical, organizational, and social systems. Ability, at least qualitatively, to understand and analyze interactions between human operators and technical systems, as well as human interrelations in organizational systems, is required. This ability is equally needed to operate optimally in a project engineering team.
Loyalty and Individual Responsibility: A proper understanding and appreciation of company goals and work ethics will be essential for the success of the corporation and each individual working for it. The roles of leadership and followers, authority, responsibility and accountability are essential elements. Sometimes leadership must be taken, but equally important is the ability to follow a decision in a loyalty to the company. These are skills and attitudes that may not be subject to learning, but an integrated system view to the functions of the corporation will help the individual to meet those requirements.

Global and Environmental Concerns: The engineering discipline is an international trade, integrated globally and with all other societal activities. The discipline must play its role and take its share of responsibility for global and environmental issues. Despite these concerns, one will hardly find Life Cycle Assessment of products and their manufacturing processes in the traditional engineering education. The same applies to the analysis of needs and their rationales, definition of performance requirements, systematic testing and evaluation, etc. However, the future trends in doing business and designing production systems will inevitably bring about greater emphasis on these issues as societies and politics change, hence be one of the greatest challenges to the engineering profession. Because the time constants and development times of education are much longer than those of the technical and even cultural issues in society are, there is an urgent need to start integrating system thinking and systems engineering into the education now.

\section{Systems Engineering Education Concepts}

The fairly general statement of professional needs stated above apply to all disciplines when the step is taken from education to professional life. In the progress through the systems engineering methodology, the search for concepts follows after the definition of requirements. There are basically four different concepts of education in systems engineering.

1) New Discipline Concepts: The idea behind this concept is to break up the traditional engineering education and introduce a new profession, all based on system theory and the wide scope of systems engineering methodology. Several schools have adopted this approach, but the results are mixed. In order to avoid a purely theoretical and abstract conceptual education, this approach should have a basis of applications, as for example operational research, industrial engineering, technology management, or in the present context, traditional engineering. This argument is again based on the experience that theories and concepts are better understood and appreciated when connected to applications. Lack of reference to practice may cause a practitioner to feel uncomfortable whenever concrete problems of an application are brought up in cooperative work and in system integration workshops.

2) Graduate Education Concepts: Another concept is to require a completed engineering degree, and build systems engineering education on top of that. The pattern of education, with examples, projects, etc., is then tailored to the engineering profession, but with systems engineering methodology. Several schools have practiced that, but have a problem with academic recognition, due to the more practical and less scientific approach to courses and projects. The professional skills in tra- 
ditional engineering are preserved, and the practitioner gets an added feeling of professional security, because previous hard core engineering problems are now better understood, and in an integrated context [2]. A problem with this concept is that it takes longer time and turns out fewer practical systems engineers.

3) Industrial Education Concept: Several companies have a pragmatic approach to systems engineering education, which is somewhat similar to the graduate concept. The idea behind this concept is that professional engineering education is good enough for industry. They observe their employees and encourage practical training in their systems engineering activity when they see interests and talents. The idea behind this again, is that not all individuals are interested or feel inclined to integrated cross-disciplinary work as systems engineering. This concept is expensive to industry and requires specific management efforts. As an average, industry has to invest at least $300 \mathrm{~h}$ in an engineer to get the person the basic systems engineering ability.

4) Integrated Engineering Concept: The idea behind this concept is to integrate systems engineering in all engineering disciplines, without sacrificing the professional quality of that education, as required internationally and by the professional organization. Integration also means integration with practical experience in the form of visiting lecturers from industry, cases from industry, etc. As it was pointed out in the education system analysis above, much may be gained by this, both for the profession and for the promotion of systems engineering. The goals and objectives of the professional education are clearer, both for faculty and students. The interaction and purpose of each subject to be taught become clearer and more logical. The project works in all disciplines, as well as the cooperative team learning use a common methodology. In short, the engineering education becomes a better preparation for professional life, to the better for both employee and employer.

\section{E. Evaluation of Systems Engineering Education Concepts}

Testing and evaluation of an educational program performance is extremely difficult, as alluded to above. The best partner in this analysis is the industry itself, the final judge of the systems engineering education performance. A practical concept, which has been proven relatively successful in systems engineering graduate programs, is an industrial advisory board [2], where members of that board may participate in teaching, in project proposal and in observation of students' performance during examinations.

Analyze System Performance: The problem with the analysis of education performance is the lack of predictive models. However, certain expected profiles of the education may be suggested, against which the education system may be measured. The actual system performance of a given sequence and contents of courses may best be evaluated retroactively from the student performance. The evaluation is complicated even further, because the students' performance is a combination of the education program and the students' ability to learn. This again is influenced by prior education experience and society culture. There is a general uncertainty principle in the observation.
Test and Evaluation of Concepts: According to the systems engineering methodology the four concepts above shall be tested against the requirements to systems engineering education, as suggested in the previous paragraph:

1) New Discipline Concepts: The main deficiency in this approach may be that it does not comply with the professional requirements, if not related to the practice of that profession. In an attempt to educate engineering generalists and system thinkers, one may lose the professionalism, whereby employment in enterprises with such requirements may be problematic. The requirement to professional security of the individual may not be met, and independent decisions may be in error. From this point of view, the concept is not recommended, unless a new type of relevant professionalism is defined for that particular education path.

2) Graduate Education Concepts: This concept does not satisfy the time requirement to engineering education; it imposes extra time and workload on the student. On the other hand, it meets the requirement of continued professional education; it meets the requirement of professional skills and knowledge, as well as professional security. It may not fit into a university requirement if a graduate school is not established. Otherwise, most of the requirements to systems engineering education are satisfied. A major disadvantage of this concept is the time requirement and the low number of professional system engineers turned out.

3) Industrial Education Concept: This concept takes the education initiative out of the university hands, and it has a serious handicap. The industrial approach may develop a subculture, which is not in touch with the academic development of the subject. Uncertainties may develop as an individual moves from one company to another. Furthermore, the academic and professional standard requirements to systems engineering may not be complied with. Furthermore, the added costs for industry is another disadvantage with this concept.

4) Integrated Engineering Concept: This concept seems to comply with most requirements, but a major problem arises. The diffusion process of systems engineering concepts, methodologies, and theories into all engineering disciplines needs a driving force. If this driving force is multivariable without any unifying denominator, the situation will remain roughly as it is today. Each discipline has its own driving force, terminology, and methodology; the advantage of a unified approach is lost. A coordinating body of system theory and systems engineering seems to be required, but this body will be different from a traditional engineering department. It shall in principle serve all departments in system theory and system thinking, in a way similar to mathematics. It may not have undergraduate programs (then we are back to alternative 1), but may have graduate programs and continuing education programs for industry and business. An added requirement to this concept is that the systems engineering concepts and processes are integrated with the specific curriculum of the engineering profession in a natural way, to avoid the perception that systems engineering is something foreign and special, with little reference to the normal engineering education.

Modify Concepts According to a Tradeoff: The iterative improvement of education shall be an integrated part of any pro- 
gram. In the following, assume concept number 4 to be accepted. The cooperation between faculty, the recognition of interdepartmental activity, as well as the cooperation with industry is now essential. This leads to additional requirements set by the various departments, industry and the university as a whole. Such requirements and the requirements to interdepartmental cooperation are known to comprise conflicting requirements. An academic advisory board [2] may take care of some of the tradeoff between those requirements, as well as serve as an academic quality control board.

Accept a Baseline Concept for Detailed System Design: The evaluation of the four concepts suggested for systems engineering education leads to a recommendation that the education is integrated into the traditional engineering education. This is the recommended baseline concept for detailed design. That design involves outline of courses, integration with existing courses, establishment of a department or a center for system theory and systems engineering, which shall serve the engineering departments in a way similar to the basic sciences, physics, mathematics, and chemistry. The detailed design shall include the total system design and performance evaluation in a concurrent fashion, life cycle assessment including cost and revenues, maintenance, integrated logistic life cycle support, changes and upgrade requirements, etc.

\section{CONCLUSION}

A short overview of system theory and systems engineering methodology has been given. On this basis is an engineering education system analyzed. The systems engineering methodology is used to present a conceptual design of an education for systems engineering. Four different concepts are evaluated against the requirements defined for the education, as seen from the various stakeholders, the students, the university, the industry, and the faculty. Based on this evaluation the pros and cons are used in a qualitative tradeoff, recommending a systems engineering education integrated in all disciplines. However, with a body of expertise (center or department) serving as the integrating denominator for systems engineering, and also serving the education process in the various engineering departments. In order to succeed with the integrated concept of systems engineering education, a major task may be to educate the normal engineering educators in system thinking and systems engineering.

However, several new professions have come out of system theory, system thinking, and systems engineering, and several traditional professions include system theory in their education. The main purpose of this paper is to emphasize the advantage of an integrated approach to engineering education, under a common set of concepts and definitions. The integration of systems engineering in all engineering disciplines, and on the premises of those disciplines, is one such integrated approach.

\section{REFERENCES}

[1] O. A. Asbjornsen, "Industrial ecology and systems engineering-A perfect match?," in Proc. 9th Annu. Int. Symp. INCOSE, Systems Engineering: Sharing Future, Brighton, U.K., June 6-10, 1999.
[2] —_ "Systems engineering at the Norwegian Technical University, Annual Report," Dept. Thermal Energy Hydropower, Norwegian Univ. Sci. Technol., Trondheim, Norway, 1995.

[3] _ - "Improved system understanding by similarities and analogies," Eng. Manage. J. Amer. Soc. Eng., vol. 4, no. 3, p. 30, 1992.

[4] _ - "Systems engineering at a university-A confrontation with old traditions and cultures," in Proc. NCOSE'91 Workshop, Los Angeles, CA, Jan. 17-18, 1991.

[5] — "Systems engineering-An educator's perspective," in Proc. 2nd Shell Process Control Workshop, D. M. Prett and C. Garcia, Eds: Butterworth Publishing, 1989, p. 489.

[6] _ - "Control and operability of process plants," Comput. Chem. Eng., vol. 13 , pp. 351-364, 1989.

[7] —, "Technical management, a major challenge in industrial competition," Chem. Eng. Progr., vol. 84, Nov. 1988.

[8] B. S. Blanchard and W. J. Fabrycky, Systems Engineering and Analysis. Englewood Cliffs, NJ: Prentice-Hall, 1990.

[9] J. Fisher, "Model-based systems engineering: A new paradigm," INSIGHT, vol. 1, no. 3, 1998 .

[10] M. A. Ottenberg and O. A. Asbjornsen, "Toward a new generation education: An application of systems engineering principles," in Systems Engineering Workplace, 3rd Annu. Int. Symp. NCOSE, W. A. McCumber and J. E. McAuley, Eds. Washington, D.C., July 26-28, 1993, pp. 337-344.

[11] N. Rescher, Cognitive Systematization. A Systems-Theoretic Approach to a Coherentist Theory of Knowledge. Oxford, U.K.: Oxford Univ. Press, 1979.

[12] M. A. Quintanilla, "Technical systems and technical progress: A conceptual framework,", http://scholar.lib.vt.edu, vol. 4, 1998

[13] H. Rombach, Substanz, System, Struktur. Die Ontologie des Funktionalismus und der philosophische Hintergrund der modernen Wissenschaften, 2nd ed. Freiburg, Germany: Alber, 1965.

[14] P. M. Senge, The Fifth Discipline-The Art and Practice of the Learning Organization. New York: Doubleday, 1990.

[15] J. Whitney, The Trust Factor-Liberating Profits \& Restoring Corporate Vitality. New York: McGraw-Hill, 1994.

Odd Andreas Asbjornsen received the M.Sc. degree in mechanical engineering in 1955 and the Ph.D. in chemical engineering in 1962, both from the Technical University of Norway, Trondheim.

He was a Full Professor with the same university from 1955 to 1982, and from 1992 to 1999, with the University of Houston, Houston, TX, from 1983 to 1986, and the University of Maryland, College Park from 1986 to 1994. He had extensive experience as a Guest Lecturer and an Industrial Consultant, in particular in the fields of process and systems engineering. He authored or co-authored over 100 papers in the international literature. He died in 1999.

Dr. Asbjornsen was an honorary member of the Norwegian and Swedish Academies of Engineering Sciences and the Royal Norwegian Society of Sciences, and received the Medal of Honor for Professional Achievement from the Norwegian Society of Professional Engineers, and an honorary doctorate from Abo Academy, Finland.

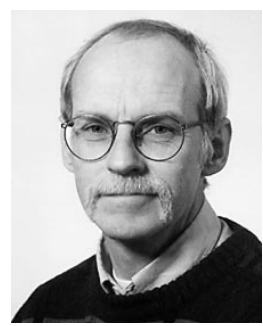

Robbert J. Hamann received an aerospace engineering education at Delft University of Technology (DUT), Delft, The Netherlands, and Princeton University, Princeton, NJ.

From 1974 to 2000, he was with Fokker Space, Leiden, The Netherlands, as an Engineer and Systems Engineer for many space projects. From 1990 to 2000, he was in charge of introducing, developing and maintaining the systems engineering methodology at Fokker Space. Since 2000, he has been a Visiting Lecturer with DUT and the University Twente, The Netherlands, and the Ecole des Mines de Nantes, France. Since February 2000, he has been Coordinator of Space Systems Engineering and Senior Lecturer at DUT. 